\title{
Perspectives of stem cell research in ophthalmology
}

\author{
Jian $\mathrm{Ge}^{1}$ \\ ${ }^{1}$ State Key laboratory of Ophthalmology, Zhongshan Ophthalmic Center, Sun Yat-sen University, Guangzhou 510060, China
}

The current stem cell research in ophthalmology focuses on the following aspects: 1) isolation, purification, and expansion of adult stem cells; 2) differentiation of embryonic stem cells, or trans-differentiation of adult stem cells, into corneal and retinal cells; 3 ) tissue engineering of ocular surface tissues by three dimensional dynamic culture system; 4) isolation and differentiation of retinal stem cells from human retinoblastomas; and 5) construction of natural animal models of eye diseases with xenografted chimeras, et al. However, problems remained unsolved in 1) signal pathway and molecular mechanism of specific differentiation from stem cell into eye cells; 2) functional reconstruction of ocular surface and retinal tissue; and 3) immune response and inflammation after stem cells transplantation. Fortunately, recent advances offer new insights for ophthalmic stem cell research: 1) establishment of pluripotent cells, from parthenogenesis or reprogramming of somatic cells to avoid ethics and immune rejection for ocular cell regeneration; 2) xenografted chimeras facilities function research of adult stem cells during eye development.

Keywords: stem cell, ophthalmology, cornea, retina

Cell Research (2008) 18:s115. doi: 10.1038/cr.2008.205; published online 4 August 2008

Correspondence: Jian Ge

E-mail: gejian@mail.sysu.edu.cn

Jian Ge, MD/PhD, is the current Vice President of Chinese Ophthalmological Society. Dr. Ge received a PhD in Ophthalmology from Sun Yat-sen University of Medical Sciences. He is current Director and Professor of Zhongshan Ophthalmic Center / State Key Laboratory of Ophthalmology, Sun Yat-sen University. He has been elected as the
President of Chinese Glaucoma Society and Vice Editor-in-Chief of Chinese Journal of Ophthalmology. Professor Ge, as the chief specialist, has won a grant from The National Basic Research Program of China (973 Program) for the research on damage and protection of optic nerves. He is the first ophthalmologist in China to be the chief specialist of this most important and prestigious program. Dr Ge's research focuses on corneal reconstruction and retinal regeneration by tissue engineering and stem cell. 